Journal of Animal and Veterinary Advances 11 (17): 3206-3212, 2012

ISSN: $1680-5593$

(C) Medwell Journals, 2012

\title{
Directed Neural Differentiation of Rabbit Umbilical Cord Mesenchymal Stem Cells
}

\author{
${ }^{1,}{ }^{3}$ Hongcheng Wang, ${ }^{2}$ Zongtao Wang and ${ }^{3}$ Hongxian Yu \\ ${ }^{1}$ Horticultural Sub-Academy, Heilongiiang Academy of Agricultural Sciences, 150069 Harbin, China \\ ${ }^{2}$ Heilongjiang Academy of Agricultural Sciences, 150086 Harbin, China \\ ${ }^{3}$ College of Wildlife Resources, Northeast Forestry University, 150040 Harbin, China
}

\begin{abstract}
Mesenchymal Stem Cells (MSC) are known to have the ability to differentiate into various lineages of mesenchymal tissue. They are widely distributed in a variety of tissues in the body and are also present in the foetal environment. In this study researchers have evaluated the possibility of MSC or cells with MSC-like potency being present in the Umbilical Cord (UC). To research the biological characteristics of rabbit Umbilical Cord Mesenymal Stem Cells (UCMSCs), the methods of immunocytochemistry and RT-PCR were used to detect the surface markers of UCMSCs. Growth curves were drawn in accordance with the cell numbers. To assess the differentiating capacity of UCMSCs, cells were induced to neural cells. These results suggested that the UCMSCs isolated from rabbit owned the similar biological characteristics with the cells separated from other species and the potential of differentiation of UCMSCs may represent an ideal candidate resource for cellular transplant therapy in tissue engineering.
\end{abstract}

Key words: Rabbit, umbilical cord, mesenymal stem cells, neural cells

\section{INTRODUCTION}

Mesenchymal Stem Cells (MSCs) can maintain self-regeneration and multi-differentiation ability in vitro and unlike embryonic stem cells, the researches of AMSCs are aloof from the ethical issues. For the reasons given above, AMSCs hold great promise for treatment of tissue repair and regeneration (Zhu et al., 2008).

Recently, Mesenchymal Stem Cells (MSCs) were found to be more effective in their differentiation potential in that they were able to give rise to many kinds of mesenchymal cells such as osteoblast, chondrocyte, tenocyte, adipocyte, cardiac myocyte, etc. (Pittenger et al., 1999; Prockop, 1997). The most commonly used source of AMSCs is the Bone Marrow (BMSCs), unfortunately, there are a few draw backs to BMSCs that are leading scientists to seek alternative stem cell sources. BMSCs are found in low numbers in tissues, tissue and organ availability is limited and BMSCs differentiation rate decreases over time (Mueller and Glowacki, 2001). In recent years, umbilical cord blood stem cells (cord stem cells) have been presented as an alternative to BMSCs. Cord stem cells are extracted from either cord blood or the Wharton's jelly of umbilical cords through enzyme digestion and have a number of critical advantages over other MSCs; the tissue is routinely discarded therefore, the tissue is available for cord stem cell extraction; the collection process of the tissue is non-invasive and there is no donor risk to an organ or tissue. (Harris et al., 2007).

In this study, researchers examined the biological characteristics of Umbilical Cord Mesenymal Stem Cells (UCMSCs) and further evaluated the feasibility of inducing the differentiation of UCMSCs into neuron-like cells in vitro. To characterize the differentiated UCMSCs, researchers examined their morphology and analyzed several markers at different passages after induction by means of RT-PCR and immunocytochemistry methods which provides a theoretical foundation and a technological method for the utilization of UCMSCs.

\section{MATERIALS AND METHODS}

Isolation of Rabbit UCMSCs: Animal experiments were performed in accordance with the guidelines established by the Institutional Animal Care and Use Committee at UMDNJ-RWJMS. The Wharton's jelly was obtained from 3 weeks old rabbit embryos under the sterile conditions, washed 3 times with PBS, removed amniotic membrane and vascular, cut in to about $1 \mathrm{~mm}^{3}$ small pieces and then digested for $15 \mathrm{~min}$ under $37^{\circ} \mathrm{C}$ using the $0.1 \%$ collagenase type IV (sigma, US). Enzymatic digestion was then neutralized with L-DMEM (Gibco, USA) supplemented with $10 \%(\mathrm{v} / \mathrm{v})$ FBS (Clontech, USA). The

Corresponding Author: Hongxian Yu, College of Wildlife Resources, Northeast Forestry University, 150040 Harbin, China 
suspension was filtered with $74 \mu \mathrm{m}$ mesh sieve and centrifuged at $200 \mathrm{~g}$ for $5 \mathrm{~min}$ at room temperature. The cell pellet was resuspended with complete medium containing L-DMEM, $10 \%(\mathrm{v} / \mathrm{v}) \mathrm{FBS}$ and $10^{4} \mathrm{IU} \mathrm{mL}^{-1}$ penicillin/streptomycin. The cell suspension was plated and incubated at $37^{\circ} \mathrm{C}$ with $5 \% \mathrm{CO}_{2}$. At $24 \mathrm{~h}$ after initial plating, the cells were washed twice with PBS to remove non-adherent cells. When the cells reached $80 \%$ confluence, trypsinization juice containing $0.25 \%$ trypsin and $0.01 \%$ EDTA (Gibco) was added to dissociate the cells from plates then trypsinization was terminated with complete medium. Cells were subcultured into new plates and after 3-4 passages, the cells were purified (Markov et al., 2007).

Immunocytochemistry for surface marker: Immunocytochemistry was performed on UCMSCs cultured for passage 3 to detect CD29, CD31, CD34, CD44, CD71, CD90 and CD105.

Rabbit UCMSCs were fixed in ice-cold ethanol and blocked in $10 \%$ bovine serum albumin for $45 \mathrm{~min}$ at room temperature. Primary antibodies against rabbit CD29, CD 31, CD34, CD44, CD71, CD90 and CD105 (1:100; Abcam USA) were applied and incubated overnight at $4^{\circ} \mathrm{C}$. Alexa Fluoro dye-conjugated goat anti-mouse antibodies (1:100; bioss China) were applied to Rabbit UCMSCs for $1 \mathrm{~h}$ at room temperature. Cells were mounted in Vector shield containing 4,6-diamidino-2-phenylindole nuclear stain (Sigma). The cells were examined using phase contrast microscope fluorescence microscope.

RT-PCR analysis: Collect cells of passage 1, 5 and 9, extract total RNA with Trizol (Invitrogen). Total RNA was reverse transcribed followed by 35 PCR cycles using RNA PCR kit Ver 3.0 (TARAKA, China). Information of gene specific primer pairs was shown in Table 1. PCR was performed in a $50 \mu \mathrm{L}$ mixture containing $10 \mu \mathrm{L} 5 \times \mathrm{PCR}$ Buffer (TARAKA, China), 28.5 $\mu \mathrm{L}$ dd $_{2} \mathrm{O}, 0.25 \mu \mathrm{L}$ Ex-Taq (TARAKA, China), $0.5 \mu \mathrm{L}$ forward and reverse primers and $1.5 \mu \mathrm{L}$ template cDNA. The cycling conditions consisted of initial $2 \mathrm{~min}$ at $94^{\circ} \mathrm{C}$. One cycle and than followed by 30 cycles of $30 \mathrm{sec}$ at $94^{\circ} \mathrm{C}$ (for denaturation), $30 \mathrm{sec}$ at $50-60^{\circ} \mathrm{C}$ (for annealing), $2 \mathrm{~min}$ at $72^{\circ} \mathrm{C}$ (for extension). PCR products were detected by $2.5 \%$ agarose gel electrophoresis.

Growth kinetic analysis of UCMSCs: Passage 1, 5 and 9 cells were used to analyze growth kinetics of UCMSCs separately. Cells were harvested and reseeded in 24 well plates with the density of $1 \times 10^{4}$ cells/well. Culture the cells for 7 days and counted 3 wells randomly each day. Then researchers plotted the cell growth curve and calculated the Population Doubling Time (PDT) according to the data (Karahuseyinoglu et al., 2007).

\section{Induced differentiation of UCMSCs into neural cells:} UCMSCs of passages 1,5 and 9 were vaccinated in a 24-well plate at $1 \times 10^{4}$ cells/well. These UCMSCs were divided into two groups when covering $80 \%$ surface of the culture plate. UCMSCs in the induction group were induced with $20 \%$ fetal bovine serum, $3 \mu \mathrm{mol} \mathrm{L} \mathrm{L}^{-1}$ $\beta$-mercaptoethanol (Sigma, USA) for $24 \mathrm{~h}$, washed thrice with PBS and then induced with serum-free medium containing 2\% Dimethyl Sulphoxide (DMSO, Sigma) and $200 \mu \mathrm{mol} \mathrm{L}{ }^{-1}$ Butylated Hydroxyanisole (BHA, Sigma). MSCs in the control group were incubated with serum medium (Woodbury et al., 2000).

The immunocytochemistry staining was the same as that mentioned above. The primary antibodies were neurofilament, neuron-specificenolase and (glial fibrillary acidic protein, GFAP) (1:100, Abcam). The secondary antibodies were conjugated with FITC, respectively. For

\begin{tabular}{|c|c|c|c|c|}
\hline Genes & Primer sequence & $\mathrm{T}\left({ }^{\circ} \mathrm{C}\right)$ & Cycle & Fragment size (bp) \\
\hline \multirow[t]{2}{*}{$\overline{C D 29}$} & F 5' CTGCCTGGGTGTCTGTGCGG 3' & & & \\
\hline & R 5' CCTGGCTGGGGCAGCTTGTC $3^{\prime}$ & 60 & 30 & 144 \\
\hline \multirow[t]{2}{*}{$C D 44$} & F 5' GAGGCTGTTGTGGGCCCTGC 3' & & & \\
\hline & R 5' GAGGCTGTTGTGGGCCCTGC 3' & 58 & 30 & 340 \\
\hline \multirow[t]{2}{*}{$C D 71$} & F 5' AAACGCGGAGCCCGAAGAGG $3^{\prime}$ & & & \\
\hline & R 5' CAGTTGCCTGATGGTGCTGGTGA $3^{\prime}$ & 59 & 30 & 132 \\
\hline \multirow[t]{2}{*}{ Neurofilament } & F 5' AGCCACGAGAAGCGCTGAGG3' & & & \\
\hline & R 5' CTGTGCTGAGCTCCGTGCCC3' & 66 & 30 & 175 \\
\hline \multirow[t]{2}{*}{ Neuro-specificenolase } & F 5' ACCTGCAGATTCGAGGGGGCA3' & & & \\
\hline & R 5' GGGCAAAGCGCCGTGTCTGA 3' & 63 & 30 & 171 \\
\hline \multirow[t]{2}{*}{$G F A P$} & F 5' ACCTGCAGATTCGAGGGGGCA 3' & & & \\
\hline & R 5' CAGGCAGCTAACCGCGAGCC $3^{\prime}$ & 66 & 30 & 147 \\
\hline \multirow[t]{2}{*}{$C D 73$} & F 5' CAGCAGCATTCCCGAAGACCCA 3' & & & \\
\hline & R 5'AGTGGACTGGCCGTAGCGGT 3' & 62 & 30 & 410 \\
\hline \multirow[t]{2}{*}{$C D 90$} & F 5' CAAGCCGGAGCACCTACCGC 3' & & & \\
\hline & R 5' ACGCGGTTCACTACGGCTGC 3' & 60 & 30 & 158 \\
\hline \multirow[t]{2}{*}{ GAPDH } & F 5' CCCGTTGCTGTCGCCCGTTC 3' & & & \\
\hline & R 5' GCCTTGACCGTGCCGTGGAA 3' & 62 & 30 & 249 \\
\hline
\end{tabular}


the control group, $0.01 \mathrm{~mol} \mathrm{~L}^{-1} \mathrm{PBS}$ was used to replace primary neurofilament, neuron-specificenolase and GFAP antibodies. Ten non-overlapped visions were randomized from induced stained cells, followed by the same data processing as that mentioned earlier.

\section{RESULTS AND DISCUSSION}

Isolation of UCMSCs: The isolated umbilical cord cells exhibited a fibroblast like phenotype in primary cell culture (Fig. 1). All these cells showed a plastic adherence in standard culture conditions.

After a few days the UCMSC expanded and the cells showed an elongated or oval/round shape with smooth borders. The cells were cultured to the fifteenth passage and showed the representative senescent appearance such as blebbing and karyopyknosis in most cells (Fig. 1d). Even more if cultured for long, the cells would shed from the plates.

Identification of UCMSCs: Researchers detected the surface antigen markers of USMSCs by immunocytochemistry staining and RT-PCR experiment. The results of immunocytochemistry staining showed that CD29, CD44, CD71, CD73, CD90 and CD105 were positive expression while the endothelial marker CD31, hemopoietic stem cell marker CD34 were negative (Fig. 2).

Growth kinetic assays of UCMSCs: The growth kinetic assays of different passages of UCMSCs were described by the drafting of growth curves. The growth curves of different passages all showed typical S shape. UCMSCs entered the logarithmic phase after about $48 \mathrm{~h}$, entered the plateau phase after $168 \mathrm{~h}$ and the decline phase at about $240 \mathrm{~h}$ later (Fig. 3). The average PDT of UCMSCs was approximately $32.75 \mathrm{~h}$.

\section{In vitro differentiation culture condition and induced} UCMSCs into neuron cells: UCMSCs were pre-induced for $24 \mathrm{~h}$. After $24 \mathrm{~h}$, the spindle shaped cells begun to contract and changed to irregular shapes (Fig. 4). Once the induction began, cell bodies further contracted and became round, triangular or cone-shaped during multipolar processes. Processes continued to elaborate, displaying many branches, growth cone-like terminal expansions. Some cells underwent a long process with evident varicosities, similar to the long axon of neuron (Fig. 4). The cell morphology became stable after $6 \mathrm{~h}$. Microscopic observation showed about 70\% cells exhibited a typical neuron-like morphology (Fig. 4). It was indicated by the immunocytochemistry staining and RT-PCR detection that non-induced UCMSCs did not express neurofilament, neuron-specificenolase and GFAP. After $6 \mathrm{~h}$ of induction, all UCMSCs in different passages expressed neurofilament, neuron-specificenolase and GFAP (Fig. 4 and 5b).

Cell therapy has emerged as a strategy for the treatment of many human diseases. At present, tissue stem cells were tentatively expanded and orientationally

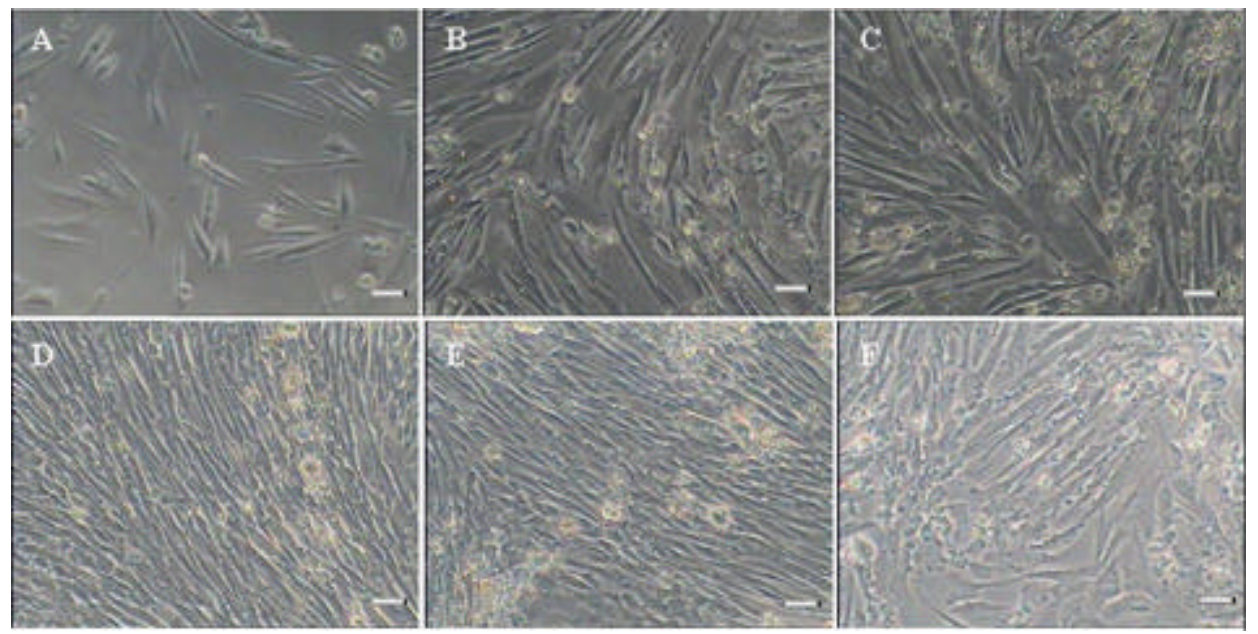

Fig. 1: Morphology of primary cultured and subcultured UCMSCs. A) Primary cells after culture for $24 \mathrm{~h}$. Many cells began to adhere and stretch. B) UCMSCs exhibited a fibroblast-like morphology and migrated easily. C) UCMSCs grew to $80-90 \%$ confluence about 5 days later and the cells were arranged in a whirl pattern. D and E) Morphology of P5 and 15 UCMSCs. After 3-4 passages, UCMSCs were purified and there were no obviously morphological differences among different passages. F) The UCMSCs of passage 22 displayed representative senescent appearances. Scale bar $=50 \mu \mathrm{m}$ 


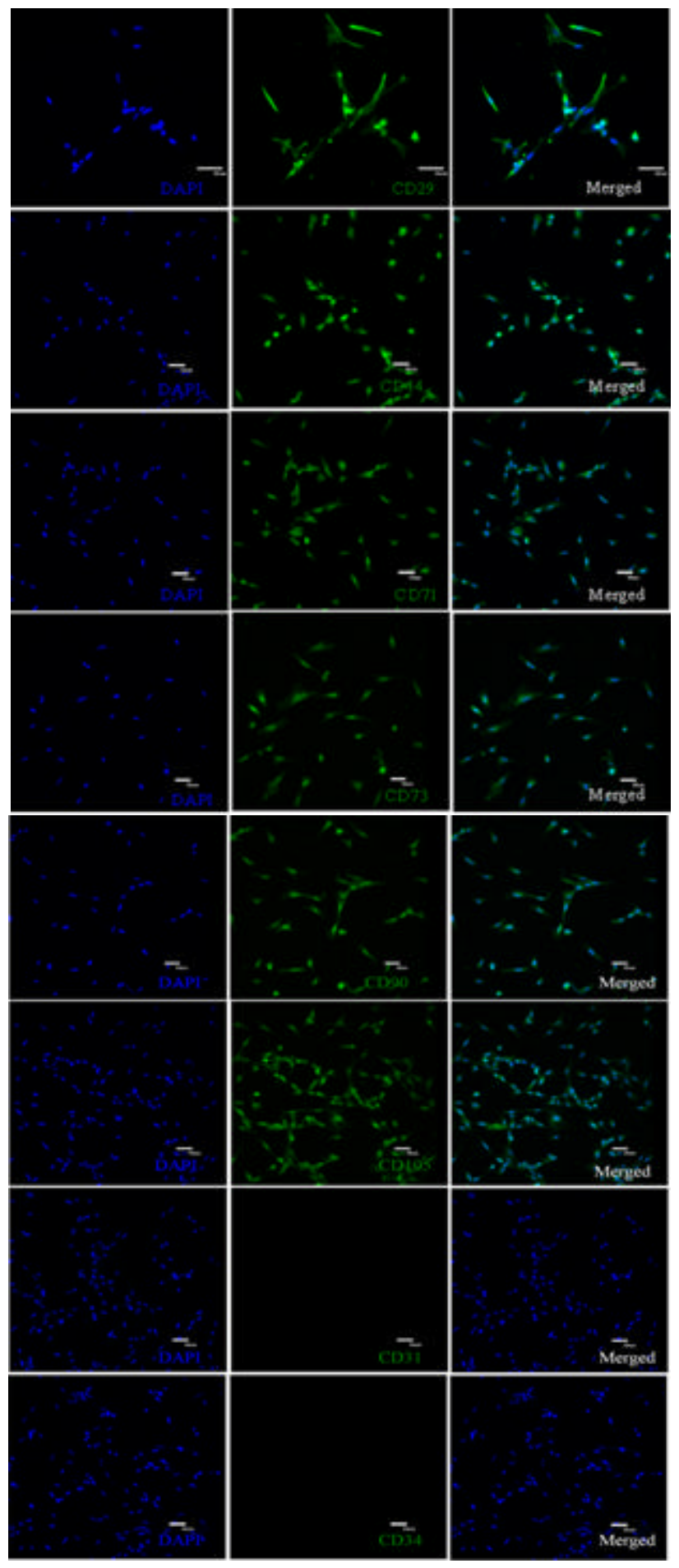

Fig. 2: Surface markers of the UCMSCs. Cell surface markers of the UCMSCs resemble with those of BMSCs, researchers detected the expression of $\mathrm{CD} 29, \mathrm{CD} 44, \mathrm{CD} 71, \mathrm{CD} 90, \mathrm{CD} 105$ and CD73 using immunocytochemistry. Immunocytochemistry showed that CD29, CD 71, CD73, CD90, CD105 and CD44 were positively expressed while CD31 and CD34 were negative. Scale bar $=50 \mu \mathrm{m}$ 
induced in vitro to some cells that are needed which are then implanted into patients to repair damage, to replace regressive tissue and improve the function of hereditarily defective tissue. The transplantation of hematopoietic stem cell has been widely used in the treatment of hematopoietic malignent diseases which provides an exemplification for the study of tissue stem cells. It was reported that MSCs could be used to repair and reconstruct some tissues such as bone cartilage, lung, brain, etc. (Pereira et al., 1998). Human MSCs cultured in vitro were implanted into rat brains (Azizi et al., 1998; Pereira et al., 1998). At present, the number of MSCs in mononuclear cells is not enough for tissue engineering. Accordingly, it is necessary to expand and purify MSCs in vitro. In this study, researchers successfully isolated the UCMSCs from umbilical cord of 3 weeks old rabbit embryos. The cell surface markers of UCMSCs resemble with BMSCs. They both express some surface markers of mesenchymal stem cell. In the research, researchers examined the expression of CD29, CD44, CD71, CD90, CD105 and CD73 by the methods of immunocytochemistry and RT-PCR. CD29 is an integrin unit associated with very late antigen receptors. It involves in cell adhesion and recognition in a variety of

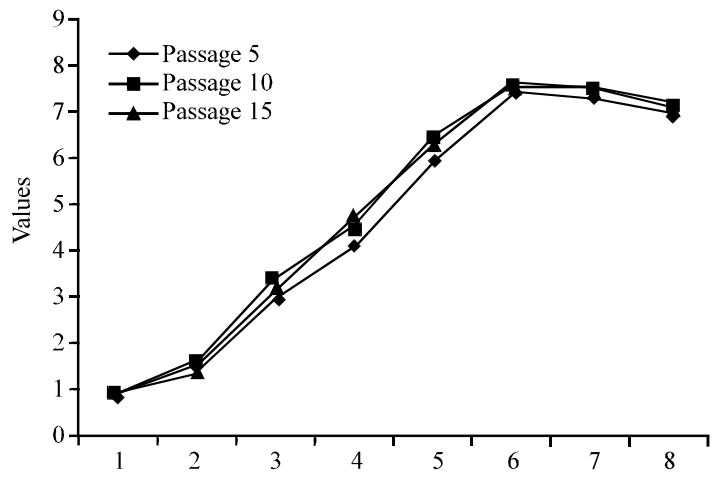

Fig. 3: Growth curves of the UCMSCs. The growth curves of P5, P15 and P20 UCMSCs were all typically sigmoidal with cell density reflected by the vertical axis. The growth curve consisted of a latent phase, a logarithmic phase and a plateau phase. PDT calculated from the growth curve was approximately $31.72,35.63,39$ and $11 \mathrm{~h}$

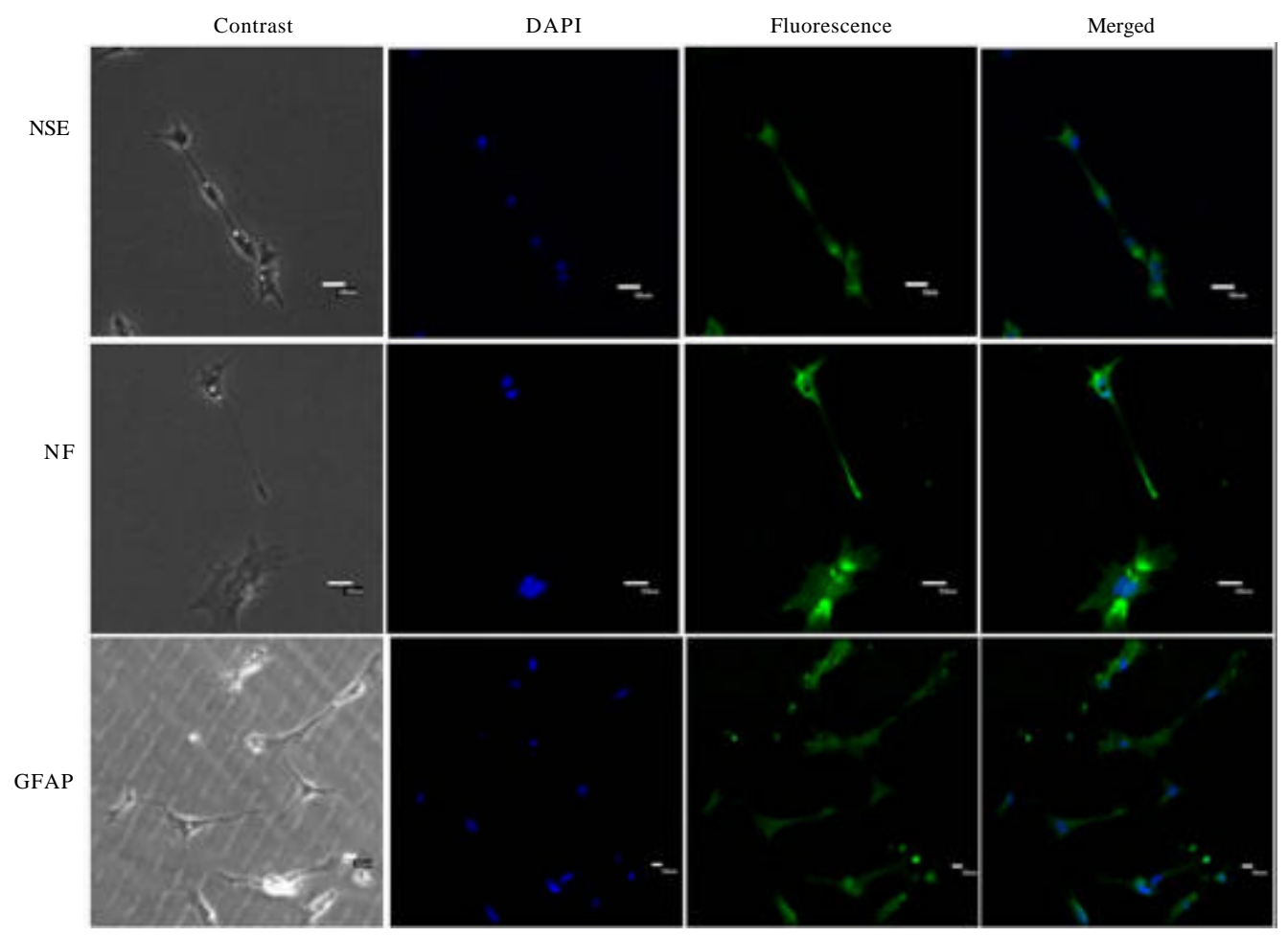

Fig. 4: Immunocytochemistry images of neurofilament, neuron-specificenolase and GFAP after the different passages of UCMSCs intervened with neural cell inducer in $6 \mathrm{~h}$. The cell morphology became stable after $6 \mathrm{~h}$. Microscopic observation showed about $70 \%$ cells exhibited a typical neuron-like morphology and expressed the neural markers. Scale bar $=50 \mu \mathrm{m}$ 


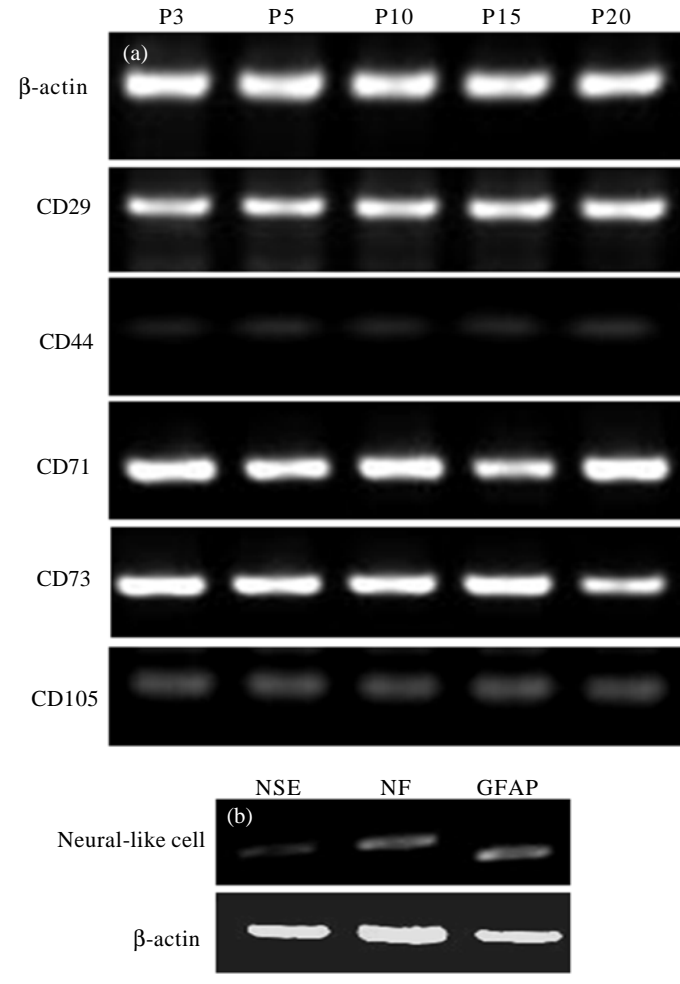

Fig. 5: Detection of UCMSC and neural cell marker by RT-PCR. a) RT-PCR analysis showed that the UCMSCs expressed CD29, CD44, CD90, CD71, CD73 and CD105. b) RT-PCR analysis showed that the neural cell expressed neurofilament, neuron-specificenolase and GFAP. $\beta$-actin in the lower picture served as internal control

processes including embryogenesis, hemostasis, tissue repair, immune response and metastatic diffusion of tumor cells. The CD44 protein is a cell-surface glycoprotein involved in cell-cell interactions, cell adhesion and migration. This protein participates in a wide variety of cellular functions including lymphocyte activation, recirculation and homing, hematopoiesis and tumor metastasis. CD71 is a member of transferring receptor family. It is needed for the transport of iron into cell and is regulated in response to intracellular iron concentration. Low iron concentration promotes increased level of transferrin receptor to take more iron into the cell.

Thus, transferrin receptor maintains cellular iron homeostasis. CD73 which is also known as 5'Nucleotidase, ecto (NT5E) is used as a marker of lymphocyte differentiation (Yan and Huang, 2006). The results suggested that UCMSCs are a group of uncommitted stem/progenitor cells differ from mesenchymal cells.
The differentiation ability of stem cells is the most notable characteristic for cell therapy. Researchers performed the differentiation of UCMSCs into neurocytes in vitro in this study. Firstly, researchers preinduced UCMSCs with L-DMEM medium containing $20 \% \mathrm{FBS}$ and $3 \mu \mathrm{mol} \mathrm{L}{ }^{-1} \beta$-mercaptoethanol and then formally induced them to differentiate into neurocytes with an induction medium composed of L-DMEM (containing 2\% DMSO, $200 \mu \mathrm{mol} \mathrm{L} \mathrm{L}^{-1} \mathrm{BHA}$ ). Both $\beta$-mercaptoethanol and BHA were antioxidants and BHA was stronger than $\beta$-mercaptoethanol (Woodbury et al., 2000). BHA and $\beta$-mercaptoethanol could assist the viability and differentiation of MSCs.

But the functional roles of BHA are not known at present. It was indicated that the optimal concentration $\left(10-50 \mu \mathrm{mol} \mathrm{L}{ }^{-1}\right)$ of $\beta$-mercaptoethanol added in a serumfree medium exhibited a prominent effect on the survival of neurons. The survival rate of neurons increased approximately from several to hundreds of folds which was related to the antioxidation of $\beta$ mercaptoethanol (Ishii, 1993).

The results indicated that UCMSCs had strong expansion capacity in vitro and differentiation potential to mesenchymal and non-mesenchymal cells and might be a new approach for cellular treatment (Woodbury et al., 2000; Ishii et al., 1993).

\section{CONCLUSION}

Researchers showed in this research that cells derived from stage 12,3 weeks old rabbit embryos exhibit a morphology and epitope profiles that are similar to those of mouse and human MSCs after extended periods in culture. The rabbit UCMSCs can differentiate into ectodermally derived neuron in vitro. This study provide evidence that with the induction of $\beta$-mercaptoethanol, BHA and DMSO, rabbit UCMSCs can also differentiate into neuron glia cells in vitro. This differentiation system could provide a simple experimental model for developing optimal cultures of neurons, astrocytes and oligodendrocytes suitable for implantation studies in animal models of PD and possible therapeutic applications.

\section{REFERENCES}

Azizi, S.A., D. Stokes, B.J. Augelli, C. DiGirolamo and D.J. Prockop, 1998. Engraftment and migration of human bone marrow stromal cells implanted in the brains of albino rats-similarities to astrocyte grafts. Proc. Natl. Acad. Sci. USA., 95: 3908-3913. 
Harris, D.T., M. Badowski, Ahmad and M.A. Gaballa, 2007. The potential of cord blood stem cells for use in regenerative medicine. Expert Opin. Biol. Therapy, 7: 1311-1322.

Ishii, D.N., 1993. Insulin and related neurotrophic factors in diabetic neuropathy. Diabet. Med. J. Br. Diabet. Assoc., 2: 14S-15S.

Ishii, K., M. Katayama, K. Hori, J. Yodoi and T. Nakanishi, 1993. Effects of 2-mercaptoethanol on survival and differentiation of fetal mouse brain neurons cultured in vitro. Neurosci. Lett., 163: 159-162.

Karahuseyinoglu, S., O. Cinar, E. Kilic, F. Kara and G.G. Akay et al., 2007. Biology of stem cells in human umbilical cord stroma: In situ and in vitro surveys. Stem Cells, 25: 319-331.

Markov, V., K. Kusumi, M.G. Tadesse, D.A. William and D.M. Hall et al., 2007. Identification of cord blood-derived mesenchymal stem/stromal cell populations with distinct growth kinetics, differentiation potentials and gene expression profiles. Stem Cells Dev., 16: 53-73.

Mueller, S.M. and J. Glowacki, 2001. Age-related decline in the osteogenic potential of human bone marrow cells cultured in three-dimensional collagen sponges. J. Cell. Biochem., 82: 583-590.
Pereira, R.F., M.D. O'Hara, A.V. Laptev, K.W. Halford and M.D. Pollard et al., 1998. Marrow stromal cells as a source of progenitor cells for nonhematopoietic tissues in transgenic mice with a phenotype of osteogenesis imperfecta. Proc. Nat;. Acad. Sci. USA., 95: 1142-1147.

Pittenger, M.F., A.M. Mackay, S.C. Beck, R.K. Jaiswal and R. Douglas et al., 1999. Multilineage potential of adult human mesenchymal stem cells. Science, 284: 143-147.

Prockop, D.J., 1997. Marrow stromal cells as stem cells for nonhematopoietic tissues. Science, 276: 71-74.

Woodbury, D., E.J. Schwarz, D.J. Prockop and I.B. Black, 2000. Adult rat and human bone marrow stromal cells differentiate into neurons. J. Neurosci. Res., 61: 364-370.

Yan, X.H. and R.B. Huang, 2006. Differentiation of human umbilical cord blood mesenchymal stem cells toward neurons induced by baicalin in vitro. Zhonghua er ke za zhi Chinese J. Pediatr., 44: 214-219, (Article in Chinese).

Zhu, Y., T. Liu, K. Song, X. Fan, X. Ma and Z. Cui, 2008. Adipose-derived stem cell: A better stem cell than BMSC. Cell Biochem. Funct., 26: 664-675. 